\title{
An Experience from a Pleural Effusion Patient: A Rare Case of Coccidioidomycosis
}

\author{
Narayana Peralaya Bailangadi ${ }^{*}$, Rayi Shripathi ${ }^{2}$, Ravi Kumar Tarikere Nagaraj ${ }^{3}$
}

1. Department of Microbiology, Lombard paramedical College and Allied Health Sciences and Hospital, Udupi, India

2. Department of Pulmonary Medicine, KVG Medical College, Sullia.India

3. Department of Microbiology, Lombard paramedical College and Allied Health Sciences and Hospital, Udupi, India

\section{ABSTRACT}

Pulmonary coccidioidomycosis is an infection in the lungs caused by the fungus Coccidioides. The disease is very rare in India and only a few cases have been reported so far. The diagnosis of pulmonary coccidioidomycosis is very challenging because it mimics other lower respiratory tract infections. Both clinical suspicion and laboratory confirmation plays a very important role in the diagnosis of this infection and in resource limited settings only culture and microscopy play a vital role in the diagnosis. We present a case of 57 years old, agriculturist and a known case of IHD, presented with pleural infection which was clinically diagnosed as tuberculosis which was later confirmed as pulmonary coccidioidomycosis. Early diagnosis helps in appropriate therapy to avoid later complications.

Keywords: Coccidioidomycosis, Lower respiratory tract infection, Pleural infection, Tuberculosis

Received: 2021/02/23; Accepted: 2021/07/10; Published Online: 2021/08/16

\begin{tabular}{|c|c|}
\hline Corresponding Information: & $\begin{array}{l}\text { Narayana Peralaya Bailangadi, , Department of Microbiology, Lombard paramedical College and Allied Health Sciences and } \\
\text { Hospital, Udupi, India. } \\
\text { Email: peralaya@gmail.com }\end{array}$ \\
\hline (c) & $\begin{array}{l}\text { This is an original open-access article distributed under the terms of the Creative Commons Attribution-noncommercial } 4.0 \text { International License which } \\
\text { redistribution of the material just in noncommercial usages with proper citation. }\end{array}$ \\
\hline
\end{tabular}

Use your device to scan and read the article online

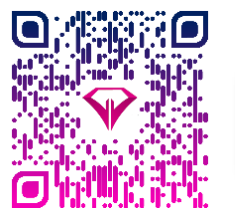

Download citation: BibTeX | RIS | EndNote | Medlars | ProCite | Reference Manager | RefWorks

$$
\text { Send citation to: } \otimes_{\text {Mendeley }} \mathrm{Z} \text { Zotero } \overline{\text { Hi RefWorks }}
$$

\section{Introduction}

The coccidioides is a dimorphic fungus, that exist both in mycelia or as spherules. Both mycelia and spherules are asexual forms $(1,2)$. The sexual form of coccidiosis has not been found. Two important species of this genus are $C$. immitis and $C$. occidioides posadasii. The identification of the two species of coccidioides is difficult, only phenotypical methods are used. Therefore, in the routine diagnostic laboratory, coccidioides are not identified at the species level. The disease is also called as valley fever, which transmitted by inhalation of arthroconidia, the infectious particles of coccidiosis species, typically during periods of dry weather. The disease incubation period is usually between 10-16 days, which the majority of immunecompetent patients who contract coccidioidomycosis will not develop any clinical symptoms or not seek medical attention (2-4). The infection usually presents with non-specific respiratory and constitutional symptoms such as fever, cough, dyspnoea or fatigue. More severe complications of this infection are respiratory failure, severe sepsis, or disseminated coccidioidomycosis. Localised pneumonia, formation of pulmonary nodules (with or without cavitation), pleural effusion or diffuse infiltrates are the severe compli- 
cations of the infection. The differential diagnosis of the infection if often difficult unless we have strong clinical and laboratory investigations $(5,6)$. The main aim of the study is a reported case with coccidioidomycosis to help in diagnosing the pulmonary infection.

\section{Case presentation}

A 57-year-old male, agriculturist by occupation from north Karnataka, presented to the Lombard memorial hospital with sudden loss of weight and appetite. He was a known case of IHD (Ischemic heart disease) with PIVD (Prolapsed intervertebral disc). After thorough physical and laboratory investigation found to have no abnormality, he was given appetite stimulant and sent home. After one month he came back with complaints of persistent weight loss of $8 \mathrm{kgs}$. His X-ray suggested left sided pleural infection, which was not present in his previous x-ray from our hospital (on the first visit). His Mantoux test and IGRA tests were positive for Tuberculosis. On clinical suspicion he was started empirically on anti-tuberculosis treatment (ATT) $2 \mathrm{EHRZ}+4 \mathrm{HER}$ regimen although pleural fluid analysis was not conclusive. Even after two months of ATT, his chest X-ray showed persistent pleural infection (Fig. 1). His pleural fluid was aspirated and sent to microbeology and pathology laboratory. He developed dry cough, nausea, fever, deglutition difficulty. The patient kept on losing weight. His laboratory findings revealed predominantly lymphocytes (i.e1000 cells per cubic millimetre,cumm), sugar 63 milligrams per decilitre $(\mathrm{mgm} / \mathrm{dL})$, protein 5.3 gram per decilitre $(\mathrm{gm} / \mathrm{dL})$ and adenosine deaminase (ADA) 105 Units per litre (U/L). Cartridge- based nucleic acid amplification test (CBNAAT) was negative for Tuberculosis. Bacteriology reports was sterile after 72 hours of incubation. Mycology culture report showed the colonies are moist, glabrous, membranous, and greyish initially, after 5 days of incubation white and cottony aerial mycelium was appeared (Figure 2). Further, the fungal wet mount preparation lactophenol cotton blue (LPCB) showed typical arthroconidiation with disjunctor cells (ghost cells) spherules suggest dimorphic fungi, $C$. immitis. The microscopically and cultural characteristics were also consistent with the $C$. immitis. After the mycology report, ATT regimen was stopped and started on azole drug (Itraconazole 200mg BID) for six weeks. After the antifungal treatment the patient's chest X-ray was normal with no pleural infection. His appetite improved and he regained his weight.

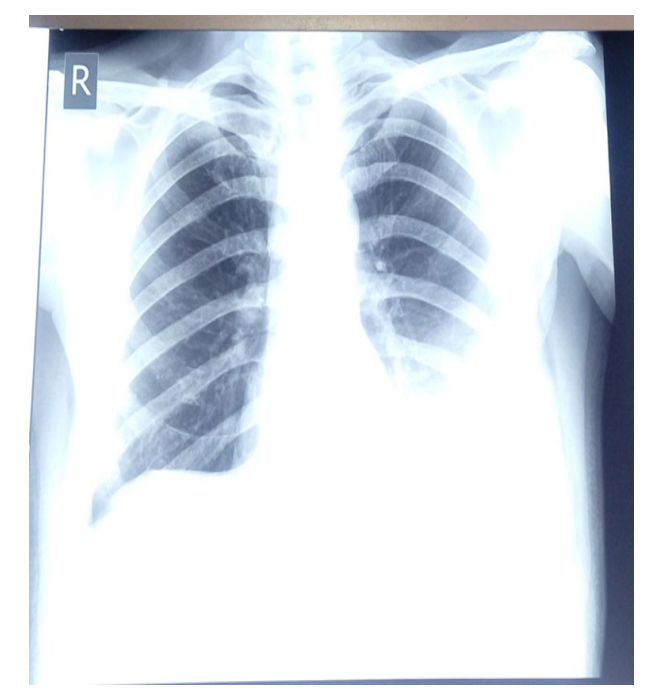

Figure 1. Chest X-ray showed Left sided pleural effusion at the presentation

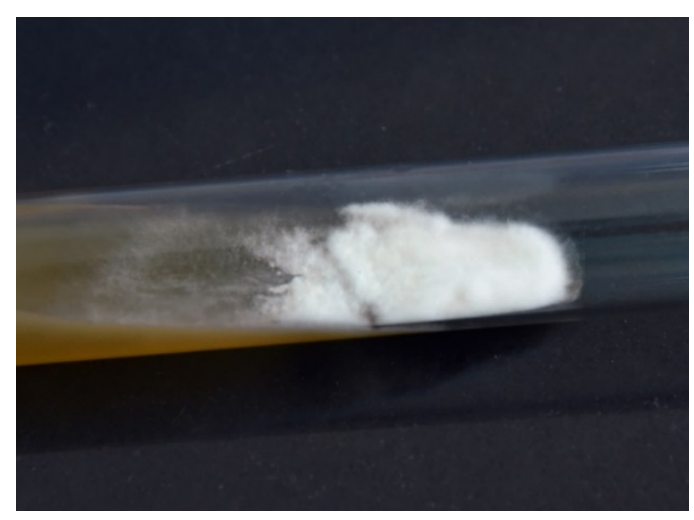

Figure 2. Cultural characteristics: Sabouraud dextrose agar (SDA) showing gray whitish floccose, aerial mycelium.

\section{Discussion}

The diagnosis of coccidioidomycosis, is often delayed. This is due to the less awareness of this condition while doing clinical diagnosis particularly in nonendemic regions (3). In the case of the patient in this study, the diagnosis was delayed because it was from a non-endemic region. This case made an impact on the clinical diagnosis and awareness. The pleural fluid analysis is actually not helpful, there is no diagnosis were made by analysis of this fluid, which may delay or exclude coccidioidomycosis $(4,5,6)$. In the case the pleural infection, analysis was usual and there was no much impact on this infection. Coccidi-oidomycosis is usually mild disease in immunocomp-etent patients and often does not require antifungal therapy $(7,8)$. Other studies have shown that imm-uno-competent patients are produce rapid and more immune response to pulmonary coccidioidomycosis, leading to increased pleural vascular permeability resulting in pleural infection formation. A fungal culture report will take 
time to report, this is the drawback of underdiagnosis of this disease $(9,10)$.

\section{Conclusion}

The microbiological and pleural fluid analysis may not differ in both fungal and tuberculous pleural infection. A simple and routine mycological culture in all cases of pleural infection may help in the appropriate treatment and also for the diagnosis. Based on the radiological findings, it is always correlated with

\section{References}

1. Rashid HR, Hunter LK. Pleural coccidioidomycosis presenting as spontaneous pneumothorax. Case Reports. 2018 Jul 27;2018: bcr-2018. [DOI:10.1136/bcr-2018-225854]

2. Jude CM, Nayak NB, Patel MK, Deshmukh M, Batra P. Pulmonary coccidioidomycosis: pictorial review of chest radiographic and CT findings. Radiographics. 2014;34(4):912-25. [DOI:10.1148/rg.344130134]

3. Tsang CA, Tabnak F, Vugia DJ, Benedict K, Chiller T, Park BJ. Increase in reported coccidioidomycosis-United States, 1998-2011. MMWR. Morbidity and mortality weekly report. 2013;62(12):217.

4. Storage TR, Segal J, Brown J. Peritoneal coccidioidomycosis: a rare case report and review of the literature. J Gastrointestin Liver Dis. 2015;24(4):527-30.

[DOI:10.15403/igld.2014.1121.244.coc]

5. Chang CC, Senining R, Kim J, Goyal R. An Acute Pulmonary Coccidioidomycosis Coinfection in a Patient Presenting with Multifocal Pneumonia With COVID-19. Journal of Investigative Medicine High Impact Case Reports. 2020 Nov; 8:2324709620972244. [DOI:10.1177/2324709620972244] the microbiological culture, which may help in rule out the differential diagnosis.

\section{Acknowledgment}

We thank Dr Sushil Jathanna, Managing Director, CSI Lombard Memorial Hospital and Group of institutions, Udupi, for providing a facility for the study.

\section{Conflict of Interest}

The authors declared no conflict of interest.

6. Pirsaraei PA, Tabsh K, Lentz J. Rare dysuria: prostatic abscess due to disseminated coccidioidomycosis. Urology case reports. 2016; 9:12-4. [DOI:10.1016/j.eucr.2016.07.008]

7. Chora-Hernández LD, Sereno-Gómez B, RuizMartínez F, Barajas-Magallon JM, Ruiz-Jiménez $M$, Corzo-Leon DE. Influenza challenging the diagnosis and management of pulmonary coccidioidomycosis. Medical Mycology Case Reports. 2020; 29:35-7. [DOI:10.1016/j.mmcr.2020.07.002]

8. Bæk O, Astvad K, Serizawa R, Wheat L, Brenøe PT, Hansen AB. Peritoneal and genital coccidioidomycosis in an otherwise healthy Danish female: a case report. BMC infectious diseases. 2017;17(1):1-4. [DOI:10.1186/s12879017-2212-4]

9. Galgiani JN, Ampel NM, Catanzaro A, Johnson RH, Stevens DA, Williams PL. Practice guidelines for the treatment of coccidioidomycosis. Clinical Infectious Diseases. 2000 Apr 1;30(4):658-61. [DOI:10.1086/313747]

10. Shah R, Shanina E, Smith RG, Bhardwaj A. A Rare Case of Disseminated Coccidioidomycosis Presenting as Brachial Plexopathy. Cureus. 2020 Nov;12(11). [DOI:10.7759/cureus.11370] 